\title{
A MORPHOLOGICAL STUDY OF PLANTARIS MUSCLE AND ITS SURGICAL PERSPECTIVE
}

\author{
Syed Nazeer Ahmed ${ }^{* 1}$, Pradeep K. Murudkar ${ }^{2}$, Md Khaleel Ahmed ${ }^{3}$.
}

${ }^{* 1}$ Assistant Professor, Department of Anatomy, KBN Medical College, Kalaburagi, Gulbarga, Karnataka, India.

${ }^{2}$ Professor and HOD, Department of Anatomy, KBN Medical College, Kalaburagi, Gulbarga, Karnataka, India.

${ }^{3}$ Assistant Professor, Department of Anatomy, KBN Medical College, Kalaburagi, Gulbarga, Karnataka, India.

\section{ABSTRACT}

Introduction: The pain is a modality of sensation given to the mankind as blessing, because unless pain is felt the person will not consult the doctor and the pathology may go un-noticed. The small fusiform plantaris muscle, with its long slender tendon, is of great importance from anatomical and from the surgical aspect. The plantaris muscle and its tendon are subject to considerable variation in both the points of origin and insertion.

Materials and Methods: Formalin fixed 50 lower limbs preserved for more than one year, approximate age between 50-60 years were dissected following the Cunningham's manual of practical anatomy, volume 01 . Origin and insertion of the muscles were studied and the muscle length and breadth measured with the Vernier's caliper and documented.

Results: The total 25 adult cadavers studied of which 23 were males and 2 were females, out of these there were 25 left and 25 right limbs. The statistical analysis was carried out by using the SPSS 19 Software After dissecting the specimens we could find insertion of the tendon is of five types and origin of the muscle belly is of six types.

Discussion: The present study carried out in 50 specimens consisting of, 46 male limbs and 4 female limbs, 25 of left side and 25 of right side. There was total $4 \%$ of bilateral agenesis in a male cadaver. Highest cases of agenesis in the review literature was by Harvey et al in the year 1983, they studied 658 lower limbs in which they found agenesis of the plantaris muscle in 126 limbs an incidence of $19.14 \%$. In the present study out of 48 limbs in which the plantaris muscle was present in 33 limbs showed the commonest type of insertion i.e. type 1 in which the tendon is directly inserted on the calcaneum medial to the Achilles tendon independently which is $68.75 \%$.

Conclusion: The agenesis of Palmaris longus is $28 \%$, and that of plantaris muscle is $10 \%$. Therefore plantaris tendon should be the tendon of choice for hand tendon repair or other reconstructive orthopedic and plastic surgeries. After going through the review of literatures by various authors, the agenesis of the plantaris muscle is not well understood. Hence further study is required for the same.

KEY WORDS: Plantaris muscle, Agenesis, Knee joint, Patellofemoral pain syndrome.

Address for Correspondence: Dr. Syed Nazeer Ahmed, Assistant professor, Department of Anatomy, KBN Medical College, Kalaburagi, Gulbarga, Karnataka, India. E-Mail: nazeer1715@gmail.com

\section{Access this Article online}

\begin{tabular}{|c|c|c|}
\hline \multirow{2}{*}{ Quick Response code } & \multicolumn{2}{|c|}{$\begin{array}{l}\text { Web site: International Journal of Anatomy and Research } \\
\text { ISSN 2321-4287 } \\
\text { www.ijmhr.org/ijar.htm }\end{array}$} \\
\hline & $\begin{array}{l}\text { d: } 12 \text { Dec } 2016 \\
\text { view: } 13 \text { Dec } 201\end{array}$ & $\begin{array}{l}\text { Acc } \\
\text { Pub }\end{array}$ \\
\hline & Revised: 02 Jan 2017 & Published (P): 31 Mar 2017 \\
\hline
\end{tabular}

INTRODUCTION

The pain in the calf has differential diagnosis, i) due to deep venous thrombosis [1-7], ii) as a complications of Achilles tendon rupture [4], 
iii) isolated plantaris tendon rupture [5-7] and also due to its role in the complicated mechanism of movements at the knee joint (because of its occasional attachment to Lateral Patellar Ligament and posterior aspect of the capsule of the knee joint) are in the list. Cruveilhier first proposed the muscle to be a vestige in man, believing that as our evolutionary ancestors assumed an erect posture, the plantaris lost its original attachment to the bottom of the foot (plantar aponeurosis) and gained a secondary attachment to the calcaneum bone of the heel [8]. The plantaris muscle is absent in only $10 \%$ of the individuals [9]. It is also called as "freshman's nerve" because of long, slenderwhite tendon which is often confused as nerve by beginners [10]. The plantaris now appears to be a highly specialized sensory muscle; the tendon serves a proprioceptive function that provides a kinesthetic sense of limb position and muscle contraction. In general, most small muscles of the body produce fine adjustments in the movement of larger muscles [11]. Many small, short muscles have been found to act across joints in parallel with much larger muscles, just like the plantaris and triceps surae.

They are named as parallel muscle combination (PMC), there believed to be nearly three dozen such PMC's in the extremities of man [12], [13]. Evolutionists continue to propose a few of our smaller and least understood muscles as being vestiges of once useful organs left over from putative evolutionary ancestors. For example, the plantaris muscle in the calf of the leg is still widely regarded to be vestigial by reason of its slender diameter and seemingly minor contribution to the two more massive muscles with which it is associated [11]. The plantaris muscle has been given little attention in the review of literature. It is most commonly mentioned only when absent from a specimen. The plantaris muscle has got highly variable anatomy like fibrous extensions to the patella, which may influence over knee function, stability, injury and rehabilitation. The agenesis of the plantaris muscle may affect the dynamics of the knee [14]. The plantaris muscle has its importance in diagnosing the pain associated conditions of the knee, because there are reported cases of unilateral double plantaris [15], firm connection of the plantaris tendon with the Achilles tendon at its mid-portion [16], and an additional tendinous origin of the plantaris muscle and its entrapment between the tibial nerve and its branch to Soleus [17].

In the "tennis leg" which is debatable since so many decades, apart from the other muscles of the leg involved, the isolated rupture of the plantaris tendon $(1.4 \%)$ is rare but as the etiology of the tennis leg has to be kept in mind for differential diagnosis when the muscle is involved [2]. The plantaris is an important muscle for the proprioceptive sense carrying to the central nervous system, also an important tendon for different types of reconstructive and other related plastic surgeries. Hence the knowledge of its tendon length, breadth and thickness will be very much useful to plan for the tendon grafting.

\section{MATERIALS AND METHODS}

The plantaris muscle was dissected in 50 adult limbs (46Male and 4Female) available in the department, the average age of the cadavers is between 50 and 60 years, the limbs were preserved in $10 \%$ formalin for more than one year. After the dissection of Popliteal fossa followed by dissection of the superficial compartment of the calf muscles by the undergraduates, careful dissection was done for the isolation of the plantaris muscle, the muscle was identified and traced from its origin to its insertion with blunt dissection and when required with the fine dissection keeping other related structures like muscles, vessels and nerves intact. The morphology and the variations in origin and insertion were noted and documented. The length, breadth and the thickness of the muscle and tendon were measured. The point of measurement taken for the muscle is from its origin to the myotendinous junction and for the tendon is from the myotendinous junction to its distal attachment. The selected photographs were taken with the Nikon coolpix digital camera.

\section{RESULTS}

The total 25 adult cadavers studied of which 23 were males and 2 were females, out of these there were 25 left and 25 right limbs each 
belonging to the same gender. The statistical analysis was carried out by using the SPSS 19 Software and the results have been tabulated and represented in the form of charts and bars. After dissecting the specimens we could find different types of origin and insertion, accordingly it is observed that the insertion of the tendon is of five types and origin of the muscle belly is of six types.

\section{TYPES OF INSERTION:}

Type-1: The tendon is inserted into the calcaneum medial to the Achilles tendon in 33 out of 48 lower extremities examined, an incidence of $\mathbf{6 8 . 7 5 \%}$ the most common type of insertion we have encountered.

Type-2: The tendon near its insertion thinned out laterally to form a fan shaped expansion which is inserted into the calcaneum superficial to the Achilles tendon in 5 out of 48 , an incidence of $\mathbf{1 0 . 4 1 \% . ~}$

Type-3: The tendon near its insertion thinned to form a fan shaped expansion which is inserted into the calcaneum deep to Achilles tendon in 2 of $48 \mathrm{limbs}$, an incidence of $4.16 \%$.

Type-4: In this type of insertion the tendon is deep to the Achilles tendon; in addition few slips from the tendon are attached to the flexor retinaculum in 2 of 48 limbs, an incidence of $4.16 \%$.

Type-5: The tendon inserted into the calcaneum along with the Achilles tendon in 6 out of 48 limbs in which the plantaris was present, an incidence of $12.5 \%$. There was complete bilateral agenesis of plantaris muscle in 2 limbs of the same male cadaver, an incidence of $\mathbf{4 . 1 6 \%}$. Table-01 shows the incidences of different types of plantaris tendon insertions.

TYPES OF ORIGIN: There is not much literature available on the variations of plantaris muscle in relation with its origin, unless as a case report which brings the attention of specially the orthopedic surgeons for the diagnosis of the patellofemoral pain syndrome (PFPS). In the present study we also encountered a case in which few fibers of the muscle are arising from the lateral patellar ligament bilaterally in a male cadaver. Depending upon the observations six types of origin of the muscle belly are described as follows;
Type-1: The muscle fibers originated from the popliteal surface of supracondylar ridge just proximal to the origin of lateral head of gastrocnemius and some fibers also from oblique popliteal ligament in 12 out of 48 limbs dissected, an incidence of $\mathbf{2 5 \%}$.

Type-2: The muscle was originated from the following sites, popliteal surface of supracondylar ridge, oblique popliteal ligament and thin bundle of fibers from the posterior part of the capsule of the knee joint in 14 of 48 limbs, an incidence of $29.16 \%$.

Type-3: From popliteal surface of supracondylar ridge of the femur and from posterior surface of lateral condyle of femur in 17 of the 48 limbs, an incidence of $\mathbf{3 5 . 4 1 \%}$, the commonest of all types of origin.

Type-4: From Supracondylar ridge, posterior surface of lateral condyle, posterior part of the capsule of the knee joint and few slips of muscle fibers originated from the lateral patellar ligament in 2 of 48 lower limbs, an incidence of 4.16\%. In this case the muscle presented was bilateral, having four sites of origin.

Type-5: From posteromedial surface of the lateral condyle of the femur only, shape of the muscle belly is triangular in 2 of 48 limbs, an incidence of $4.16 \%$.

Type-6: Supracondylar ridge and interdigitations with lateral head of gastrocnemius in the right limb of a male cadaver in 1 of 48 limbs, an incidence of $\mathbf{2 . 0 8 \%}$.

\section{DISCUSSION}

The plantaris muscle has been given little attention in the reviewed literature. It is most commonly mentioned only when absent from a specimen. The muscle has been phylogenetically considered as vestigial. Any structure or organ is said to be vestigial when it is degenerated or lost its functions due to evolutionary changes. Cruveilhier was the first to describe the muscle to be a vestige in man, believing that, as our evolutionary ancestors assumed an erect posture, the plantaris lost its original attachment to the bottom of the foot (plantar aponeurosis) and gained a secondary attachment to the calcaneum bone of the heel $[8,18]$. Due to progressive change of the posture, the 
human foot has become man's sole organ of support [19], and due to special development of the calcaneal protuberance, the tendon of the plantaris was pulled away from the aponeurosis and forced to seek a new secondary point of calcaneal anchorage [20]. Daseler and Anson in 1943 highlighted the importance of the plantaris muscle both from the anatomical and phylogenetic points of view and also from the surgical aspect. In American brown bear and apes the tendon passes beneath the calcaneum to blend with the plantar aponeurosis [21].

Contrary with the concept of vestigial; David N Menton in $\mathbf{2 0 0 0}$ have focused on the same and with a detail study concluded that many scientists have ignored the claims of evolutionists regarding vestigial organs, and thus the advance of empirical science has revealed at least one known function for nearly every type of organ, tissue, and cell of the body. Of the nearly 200 vestigial organs once claimed to exist in the human body, only a few continue to be seriously discussed as vestigial. In general, most small, short muscles of the body produce fine adjustments in the movement of larger muscles. One of the problems with the whole concept of vestigial or functionless muscles is the well-known fact that unused muscles quickly degenerate. People ranging from astronauts exposed to a prolonged weightless environment, to those confined to long bed rest, lose a significant amount of muscle mass in only a few months. In short, muscle mass is a matter of 'use it or lose it'. The human plantaris belly length is only about one third that of the gastrocnemius, but for any contraction or extension of this PMC, both will undergo similar changes in absolute length. Thus the plantaris will experience two to three times greater change in both relative length and rate compared to the gastrocnemius [11].

Present study carried out in 50 specimens consisting of, 46 male limbs and 4 female limbs, 25 of left side and 25 of right side. There was total $4 \%$ of bilateral agenesis in a male cadaver, which is coinciding with the study conducted by Kudo T in Japanese population in the year 1916 [22]. The agenesis found by the author was irrespective of sex and lateralization. Highest cases of agenesis in the review literature was by Harvey et al in the year 1983, they studied 658 lower limbs in which they found agenesis of the plantaris muscle in 126 limbs an incidence of $19.14 \%$ [23]. In 1994 by Osny et al [24], in 2010 by Aragao et al in 20 limbs [25] and in the year 2011 by Maayke et al in 107 limbs [16] they all observed that the plantaris muscle was identified in all the specimens. In this study out of 48 limbs in which the plantaris muscle was present 33 limbs showed the commonest type of insertion i.e. type 1 in which the tendon is directly inserted on the calcaneum medial to the Achilles tendon independently which is $68.75 \%$ Fig 1.

Fig. 1: Showing type 1of insertion on left limb in a male cadaver.

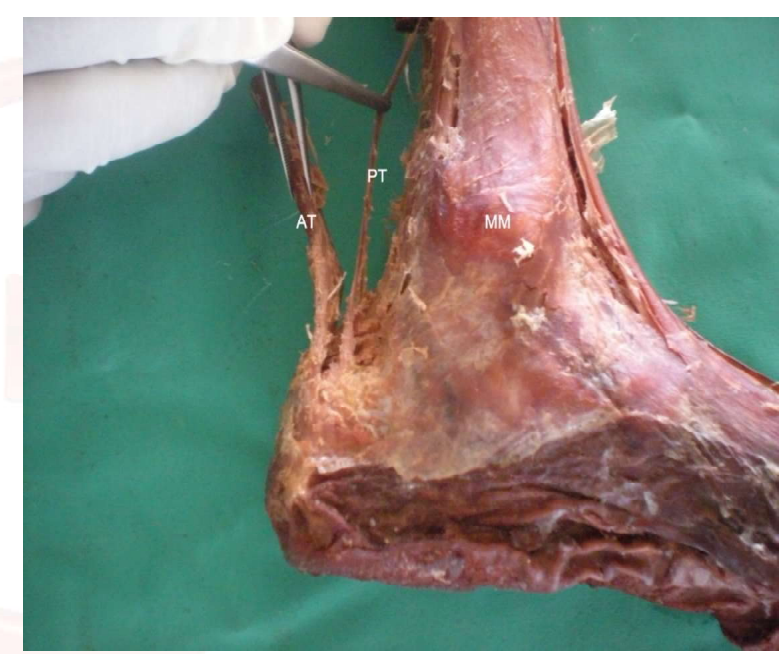

When referring to previous authors like Daseler and Anson in 1943 [21] and Schlicht S M in 1992 [26] showed that in at least $80 \%$ of their dissected lower limbs the insertion of the plantaris tendon is directly inserted to the calcaneus independent of the tendocalcaneus. In a study conducted by Nayak et al in 2010 on 52 lower limbs of 26 males have observed $36.53 \%$ of insertions of the plantaris muscle were independently to the calcaneum [27]. The standard text books like Hollinshead 1958 and Gray's Anatomy 40 th Edn 2008 have mentioned the length of the muscle belly is $7-10 \mathrm{~cm}$. The total mean length of the muscle belly in our study is $7.99 \mathrm{cms}$ which is similar to those described by the standard books. The total mean length of the tendon in this study is found to be $33.16 \mathrm{~cm}$ which is coinciding with the study conducted by Aragao J A et al in 2010 [25] and with a difference of $2 \mathrm{~cm}$ with Surut et al in 2002 [19]. 
Table 1: Showing the mean values of length, breadth and thickness of the plantaris muscle and tendon in the male and female in centimeters.

\begin{tabular}{|c|c|c|c|c|c|c|}
\hline SEX & ML cm & MB cm & MT cm & TL cm & TB cm & TT cm \\
\hline FEMALE & 7.55 & 1.35 & 0.33 & 30.72 & 0.31 & 6.75 \\
\hline MALE & 8.03 & 1.64 & 0.42 & 33.37 & 0.3 & 6.78 \\
\hline TOTAL & 7.99 & 1.62 & 0.41 & 33.16 & 0.3 & 6.78 \\
\hline SD & 2.11 & 0.58 & 0.28 & 7.16 & 0.13 & 7.24 \\
\hline
\end{tabular}

As such much literature is not available regarding the measurement of the tendon and the muscle belly, hence the work of the above two authors is taken for comparison. A very less percentage but clinically very important variation was observed in the present study i.e. the muscle originated from four sites those are; From Supracondylar ridge, posterior surface of lateral condyle of femur, posterior part of the capsule of the knee joint and few slips of muscle fibers from the lateral patellar ligament in 2 of 48 lower limbs, an incidence of $4.16 \%$ Fig 2.

Fig. 2: Showing type 4 origin on right side of a male cadaver.

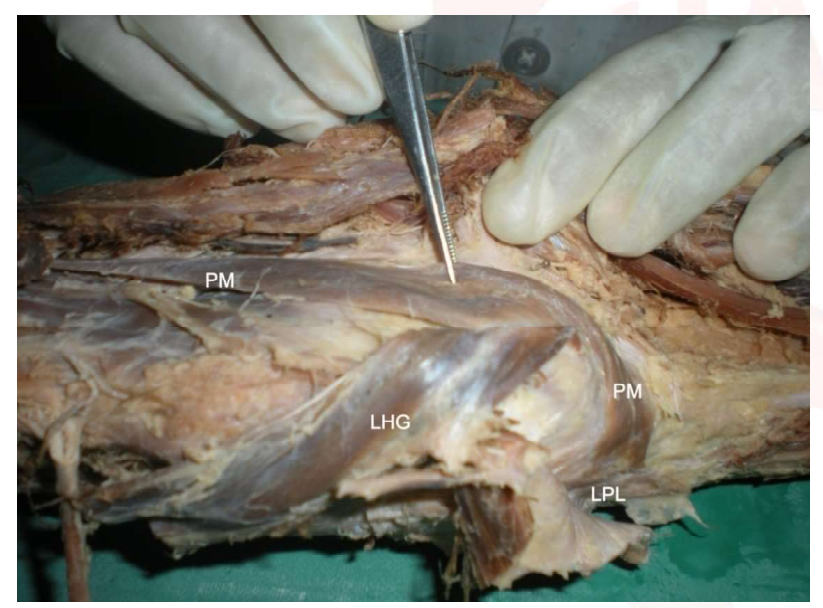

This particular variation is observed bilaterally in one adult male cadaver. This type of origin is also reported only by Freeman A. J et al 2008 in 5 of 46 cadaveric knees, an incidence of $10.87 \%$ and also 9 cases of interdigitations with the lateral head of gastrocnemius, an incidence of $19.6 \%$ [14], but in this study it is found to be $2 \%$ only.

The above types of variations may influence the knee function and stability, consequently allowing the plantaris muscle to have a varying roles in knee dynamics, injury and rehabilitation. The muscles contributing to PFPS include quadriceps, hamstrings, triceps surae, and tensor fascia lata muscles. Common causes of PFPS are asymmetrical balance of the muscles and tendons attaching to the patella that result in medial or lateral displacement [28], hence the plantaris originating from LPL which is the extension of tendon of vastus lateralis, will affect the knee dynamics. There are reported cases of unilateral double plantaris [15], bilateral double plantaris muscle [29] additional tendinous origin and entrapment of the plantaris muscle [17] and also a firm connection between plantaris and calcaneal tendon at the level of mid-portion of the Achilles tendon in $10 \%$ of specimens in a recently published article, the incidence of firm connection between the two tendons was more in the frozen cadavers without formalin, they infer that the formalin may alter the texture of the muscles hence the incidence is more [16]. In the present study the above said types of variations are not observed. All the specimens presented with plantaris muscle were having single muscle only with no double or accessory muscle. The myotendinous junction was found at the level of origin of the soleus muscle which coincides with the findings of Helms et al 1995 [5]. In all the limbs studied the nerve supply to the plantaris muscle was by tibial nerve only.

\section{CONCLUSION}

The plantaris muscle and its tendon are subject to considerable variation in both the points of origin and insertion. In the present study of 50 lower extremities, six types of origin and five types of insertions of the tendon were observed and compared with the previous studies. The agenesis of the plantaris muscle was seen bilaterally of a male cadaver, an incidence of 4 percentage. The agenesis of palmaris longus is $28 \%$ [30] and that of plantaris muscle is $10 \%$ [9], therefore plantaris tendon should be the tendon of choice for hand tendon repair or other reconstructive orthopedic and plastic surgeries. Interdigitations with the lateral head of gastrocnemius $(2.1 \%)$ and extension of few muscle fibers to the lateral patellar ligament $(4.16 \%)$ encountered in the present study, are less frequent but important variations to be considered for differential diagnosis in PFPS related pains and also these type of variations may have a more important role in knee dynamics than previously thought and thus a greater role in knee injuries. When the patient presents with 
calf muscle pain that has to be thoroughly investigated by MRI to rule out isolated plantaris rupture as it mimic deep venous thrombosis and the presentation may be that of tennis leg also. The surgeons specially should be aware of the variations of the plantaris muscle like agenesis, double plantaris, accessory tendon, and sometimes the aponeurotic expansion inserted superficial to Achilles tendon being vulnerable to injury presenting as Achilles rupture. As the plantaris tendon is a tensile and stretchable structure, it is used as a strong reinforcement membrane after suturing the partial rupture of the Achilles tendon, how far it can be useful for tympanoplasty, clinical trials may be carried out in future by the concerned ENT surgeons.

Maayke et al 2011 [16] are of view that, the future work on the plantaris muscle can be done in frozen specimens in addition to embalmed specimens to get the clarity of the attachment of the tendon. Further it is suggested that the study can be carried out in the living by using advance methods of investigations like MRI, scanning and electromyography techniques in various movements of the knee and ankle joints in collaboration with the Departments of Physiology, Orthopedics, Radiology, and the Sports medicine persons, to understand the function and its role in producing PFPS and also to take more advantage of this slender muscle in surgical grafting and other plastic surgeries. After going through the review of literatures by various authors, the agenesis of the plantaris muscle is not well understood. Hence further study is required for the same.

\section{ABBREVATIONS}

PMC - Parallel Muscle Combination

SM - Soleus Muscle

PM - Plantaris Muscle

AT - Achilles tendon

PT - Plantaris tendon

LHG - Lateral head of gastrocnemius

MM - Medial malleolus

LPL - Lateral patellar ligament

FR - Flexor retinaculum

PFPS - Patellofemoral pain syndrome

ML - Muscle length

MB - Muscle breadth
MT - Muscle thickness

TL - Tendon length

TB - Tendon breadth

TT - Tendon thickness

SD - Standard deviation

$\mathrm{mPa}$ - Milli Pascal

\section{Conflicts of Interests: None}

\section{REFERENCES}

[1]. Leekam RN, Agur AM, McKee NH. Using Sonography to Diagnose Injury of Plantaris Muscles and Tendons. AJR 1999;172:185-189.

[2]. Delgado GJ, Chung CB, Lektrakul N, Azocar P, Botte MJ, Coria D, Bosch E, Resnick D. Tennis Leg: Clinical US Study of 141 Patients and Anatomic Investigation of Four Cadavers with MR Imaging and US. Radiology 2002;224:112-119.

[3]. Gilbert TJ, Bullis BR, Griffiths HJ. Tennis Calf or Tennis leg. Orthopedics 1996;19(2):182 184.

[4]. Steenstra F, Van Dijk CN. Achilles tendoscopy. Foot ankle clin. 2006;11:429-438.

[5]. Helms CA, Fritz RC, Garvin GJ. Plantaris Muscle injury: Evaluation with MR Imaging. Radiology 1995;195:201-203.

[6]. Allard, J.C: Bancroft, J \& Porter, G. Imaging of plantaris muscle rupture. Clin. Imag.,16: 55-58, 1992.

[7]. Hamilton W, Klostermeier T, Lim E, et al. Surgically Documented rupture of the Plantaris muscle: a case Report and literature review. Foot Ankle Int. 1997;18(8):522-523.

[8]. Cruveilhier, Jean: Anatomie Descriptive. Tome II. Paris, Bechet Juene, 1834.

[9]. Standring Susan. Gray's Anatomy. The Anatomical Basis of Clinical Practice. 40 ${ }^{\text {th }}$ Edn. Elsevier Churchill Livingstone, Philadelphia, 2008. pp. 1421.

[10]. Moore KL, Dalley AF, eds. Clinically Oriented Anatomy. 5th ed. Philadelphia: Lippincott Williams \& Wilkins, 2006; 648-649.

[11]. David. N. Menton; The plantaris and the question of vestigial muscles in man. CEN Tech. J 14(2): 50-53, 2000.

[12]. Nitz, A. and Peck, D., Comparison of muscle spindle concentrations in large and Small human epaxial muscles acting in parallel combinations, The American Surgeon 1986;52:273-277.

[13]. Peck, D., Buxton D.F. and Nitz, A., A comparison of spindle concentrations in large and small muscles acting in parallel combinations, J.Morphology 1984;180:243-252.

[14]. A. Jay Freeman, Nathan A. Jacobson and Quentin A. Fogg. Anatomical variations of The plantaris muscle and a potential role in patellofemoral pain syndrome: Clinical Anatomy. 2008;21:178-181.

[15]. David M. Kwinter, James P. Lagrew; Julie, Kretzer, Cara Lawrence, Diksha Malik, Megan Mater \& Jennifer K. Brueckner. Unilateral Double Plantaris Muscle: A Rare Anatomical variation; Int. J. Morphol 2010;28(4):1097-1099. 
[16]. Maayke N. van sterkenburg, Gino M. M. J. Kerkhoffs, Roeland P. Kleipool and C.Neik Van Dijk: The plantaris tendon and a potential role in mid-portion Achilles tendinopathy: an observational anatomical study. J. Anat. 2011;218:336-341.

[17]. Nayak SR, Krishnamurthy A, V Prabhu, Madhyastha $S$; Additional tendinous Origin And entrapment of the plantaris muscle. Clinics: 2009;64(1):67-68.

[18]. Keith, A. The history of the human foot and its bearing on orthopaedic practice. J. Bone Joint Surg. Am., 1929;11:10-32.

[19]. Surut. J, Ekamol. T, Weerachai. K, Kimaporn. K. Palmaris longus and plantaris tendon: Anatomical variations and relationship, Srinagarind Med. J., 2002;17(3):160-163.

[20]. Henle J. Handbuch der Systematischen Anatomie des Menschen. Abth. Muskellehre. Braunschweig, F. Vieweg u. Sohn. 1871.

[21]. Daseler, E.H. and Anson, B.J., The plantaris muscle, an anatomical study of 750 specimens, J. Bone Joint Surgery 1943;25(4):822-827.

[22]. Kudo, T. On the aponeurosis plantaris In Japanese.. Hokuetsu Igakkai Zasshi. 1916;31:98-107.

[23]. Harvey FJ, Chu G, Harvey PM. Surgical availability of the plantaris tendon. J Hand Surg Am 8: 1983;243247.
[24]. Osny, S, Carvalho, Jr. A. E, Fernandes, T. D, Romano, D, Adachi, P. P, Neto, R. S. Musculo solear acessorio: aspectos clinicos e achados cirurgicos. Rev. Bras. Ortop, 1994;29(4):251-55.

[25]. Aragao, J. A.; Reis, F. P.; Guerra, D. R. \& Cabral, R. H. The occurrence of the Plantaris muscle and its muscle-tendon relationship in adult human cadavers. Int. J. Morphol., 2010;28(1):255-258.

[26]. Schlicht SM, Morrison WA. The plantaris tendon as a tendo-osseous graft. Part I. An anatomical study. J Hand Surg. 1992;17B:467-470.

[27]. Nayak SR, Krishnamurthy $A$, Ramanathan L, Ranade AV, Prabhu LV, Jiji PJ, Rai R, Chettiar GK, Potu BK, Clin Ter. 2010;161(3):249-252.

[28]. Callaghan M, Oldham JA. Quadriceps atrophy: To what extent does it exist in patellofemoral pain syndrome? Br J Sports Med 2004;38:295-299.

[29]. Rana KK, Das S, Verma R. Double plantaris muscle: A Cadaveric study Clinical importance. Int J Morphol. 2006;24:495-98.

[30]. K. Devi Sankar, P. Sharmila Bhanu, Susan P. John: Incidence of agenesis of Palmaris Longus in the Andhra population of India; Indian Journal of Plastic Surgery. Jan-Apr 2011;44(1):134.
How to cite this article:
Syed Nazeer Ahmed, Pradeep K. Murudkar, Md Khaleel Ahmed. A
MORPHOLOGICAL STUDY OF PLANTARIS MUSCLE AND ITS
SURGICAL PERSPECTIVE. Int J Anat Res 2017;5(1):3560-3566. DOI:
10.16965/ijar.2016.506 